\title{
Bile Acid Profiles in Peroxisomal 3-Oxoacyl-Coenzyme A Thiolase Deficiency
}

\author{
Peter T. Clayton, ${ }^{\star}$ Ella Patel, ${ }^{\star}$ Alexander M. Lawson, ${ }^{\ddagger}$ Robert A. Carruthers, ${ }^{\star}$ and Janna Collins ${ }^{5}$ \\ ${ }^{*}$ Department of Child Health, Institute of Child Health, London, WC1N 1EH United Kingdom; ${ }^{\ddagger}$ Clinical Mass Spectrometry, Clinical \\ Research Centre, Harrow, United Kingdom; ${ }^{\S}$ Department of Pediatrics, Albert Einstein College of Medicine, Bronx, New York 10461
}

\begin{abstract}
Fast atom bombardment mass spectrometry and gas chromatography-mass spectrometry were used to analyze bile acids in the body fluids of an infant (L.C.) whose liver contained no immunoreactive peroxisomal 3-oxoacyl-CoA thiolase. The profiles were compared with those of six patients with undetectable peroxisomes (Zellweger syndrome) and two siblings (N.B. and I.B.) whose defect of peroxisomal $\beta$-oxidation could not be localized by morphological studies of peroxisomes or by immunoblotting of peroxisomal $\beta$-oxidation proteins. $3 \alpha, 7 \alpha, 12 \alpha$-Trihydroxy-5 $\beta$-cholestan-26-oic acid (THCA) was present in bile and plasma of all patients. However, bile from L.C., N.B. and I.B. contained unconjugated varanic acid $(3 \alpha, 7 \alpha, 12 \alpha, 24$-tetrahydroxy-5 $\beta$-cholestan-26-oic acid) as the major $\mathrm{C27}$ bile acid, whereas bile from Zellweger patients contained only small amounts of varanic acid. In the bile from L.C. two isomers of varanic acid were present; in the bile from N.B. and I.B. a single isomer predominated. L.C., N.B., and I.B. all produced bile containing small amounts of (24E)$3 \alpha, 7 \alpha, 12 \alpha$-trihydroxy-5 $\beta$-cholest-24-en-26-oic acid ([24E]$\triangle 24-T H C A)$, its [24Z]- isomer, $3 \alpha, 7 \alpha, 12 \alpha$-trihydroxy-5 $\beta$-cholest-23-en-26-oic acid and $3 \alpha, 7 \alpha, 12 \alpha$-trihydroxy-27-nor-5 $\beta$ cholestan-24-one. The results provide evidence for peroxisomal pathways for cholic acid synthesis in man via THCA, $\triangle 24-T H C A$ and varanic acid and show that bile acid analyses can be used to diagnose peroxisomal thiolase deficiency. (J. Clin. Invest. 1990. 85:1267-1273.) peroxisomal disorders - synthesis of bile acids - beta-oxidation - pseudoZellweger syndrome $\bullet$ bile alcohols $\bullet$ mass spectrometry
\end{abstract}

\section{Introduction}

Chenodeoxycholic acid and cholic acid are synthesized from cholesterol by a series of reactions involving modifications to the steroid nucleus and oxidation of the side chain (1). The major pathway for cholic acid synthesis proceeds via the 27carbon bile acid- $3 \alpha, 7 \alpha, 12 \alpha$-trihydroxy-5 $\beta$-cholestan-26-oic acid (trihydroxycoprostanic acid, THCA). ${ }^{1}$ Oxidation of

Address reprint requests to Dr. Clayton, Department of Child Health, Institute of Child Health, 30 Guilford St., London WCIN 1EH, United Kingdom.

Received for publication 24 July 1989 and in revised form 17 November 1989.

1. Abbreviations used in this paper: CRM, cross-reacting material; FAB-MS, fast atom bombardment mass spectrometry, GC(-MS) gasliquid chromatography (-mass spectrometry); VLCFA, very long chain

J. Clin. Invest.

(c) The American Society for Clinical Investigation, Inc. $0021-9738 / 90 / 04 / 1267 / 07 \$ 2.00$

Volume 85, April 1990, 1267-1273
THCA to cholic acid occurs in the hepatic peroxisomes (2) and is thought to proceed via a series of reactions similar to the peroxisomal $\beta$-oxidation of fatty acids (Fig. 1). The first enzyme in the peroxisomal $\beta$-oxidation sequence is an acyl-CoA oxidase that introduces the $\mathrm{C} 2-\mathrm{C} 3$ double bond. The second and third steps of peroxisomal $\beta$-oxidation are both catalyzed by a "bifunctional protein," which has enoyl-CoA hydratase and 3-hydroxyacyl-CoA dehydrogenase activity. Finally a peroxisomal 3-oxoacyl-CoA thiolase splits off acetyl-CoA $(3,4)$. The oxidation of THCA to cholic acid in the peroxisomes is thought to occur in an analogous fashion to the $\beta$-oxidation of fatty acids (as shown in Fig. 1) via the coenzyme A esters of $3 \alpha, 7 \alpha, 12 \alpha$-trihydroxy-5 $\beta$-cholest-24-en-26-oic acid $(\Delta 24$ THCA), $3 \alpha, 7 \alpha, 12 \alpha, 24$-tetrahydroxy-5 $\beta$-cholestan-26-oic acid (24-hydroxy-THCA or varanic acid) and $3 \alpha, 7 \alpha, 12 \alpha$-trihydroxy-24-oxo-5 $\beta$-cholestan-26-oic acid (24-oxo-THCA). Similar intermediates are considered to participate in the peroxisomal pathway for conversion of $3 \alpha, 7 \alpha$-dihydroxy- $5 \beta$-cholestanoic acid (dihydroxycoprostanic acid, DHCA) to chenodeoxycholic acid (1). It should be emphasized, however, that there is only limited evidence for these pathways in man. Swell et al. showed that varanic acid could be converted to cholic acid in man but it was a poorer precursor of cholic acid than THCA (5).

The study of patients with peroxisomal defects has contributed considerably to our understanding of peroxisomal biochemistry. Patients with Zellweger syndrome lack normal peroxisomes; as a result they cannot oxidize very long chain fatty acids (VLCFA) and show impaired conversion of THCA to cholic acid $(6,7)$. In 1986, Goldfischer et al. described a patient with clinical, biochemical, and pathological features similar to those seen in Zellweger syndrome. Peroxisomes were present in the patient's liver but nonetheless there was an abnormal accumulation of VLCFA and THCA (8). Subsequently, Schram et al. showed that peroxisomal $\beta$-oxidation was impaired due to CRM -ve peroxisomal thiolase deficiency; an antibody to the thiolase detected no immunoreactive protein (cross-reacting material, CRM) in the liver (9). Schram et al.

fatty acids (> C22). Bile acids, alcohols, and sterols: THCA (trihydroxycoprostanic acid), $3 \alpha, 7 \alpha, 12 \alpha$-trihydroxy-5 $\beta$-cholestan-26-oic acid; $\Delta 23$-THCA and $\Delta 24$-THCA indicate $3 \alpha, 7 \alpha, 12 \alpha$-trihydroxy- $5 \beta$ cholest-23-en-26-oic and -24-en-26-oic acids respectively; varanic acid or 24-hydroxy-THCA, $3 \alpha, 7 \alpha, 12 \alpha, 24$-tetrahydroxy-5 $\beta$-cholestan26-oic acid; $1 \beta$-hydroxy-, $6 \alpha$-hydroxy-, and 25 -hydroxy-THCA indicate $1 \beta, 3 \alpha, 7 \alpha, 12 \alpha$-tetrahydroxy-, $3 \alpha, 6 \alpha, 7 \alpha, 12 \alpha$-tetrahydroxy-, and $3 \alpha$, $7 \alpha, 12 \alpha, 25$-tetrahydroxy- $5 \beta$-cholestan-26-oic acids respectively; 26hydroxy-THCA, $3 \alpha, 7 \alpha, 12 \alpha, 26$-tetrahydroxy-5 $\beta$-cholestan-27-oic acid; 24-oxo-THCA, $3 \alpha, 7 \alpha, 12 \alpha$-trihydroxy-24-oxo-5 $\beta$-cholestan-26oic acid; DHCA (dihydroxycoprostanic acid), $3 \alpha, 7 \alpha$-dihydroxy$5 \beta$-cholestan-26-oic acid; $\triangle 24$-DHCA, $3 \alpha, 7 \alpha$-dihydroxy- $5 \beta$-cholest24-en-26-oic; 24-hydroxy-DHCA, $3 \alpha, 7 \alpha, 24$-trihydroxy-5 $\beta$-cholestan26-oic acid; C29-dicarboxylic acid, $3 \alpha, 7 \alpha, 12 \alpha$-trihydroxy-5 $\beta$-27a, $27 \mathrm{~b}$-dihomo- $5 \beta$-cholestan-26,27b-dioic acid; $5 \beta$-ranol, 27 -nor-5 $\beta$ cholestan-3 $\alpha, 7 \alpha, 12 \alpha, 24,26$-pentol. 

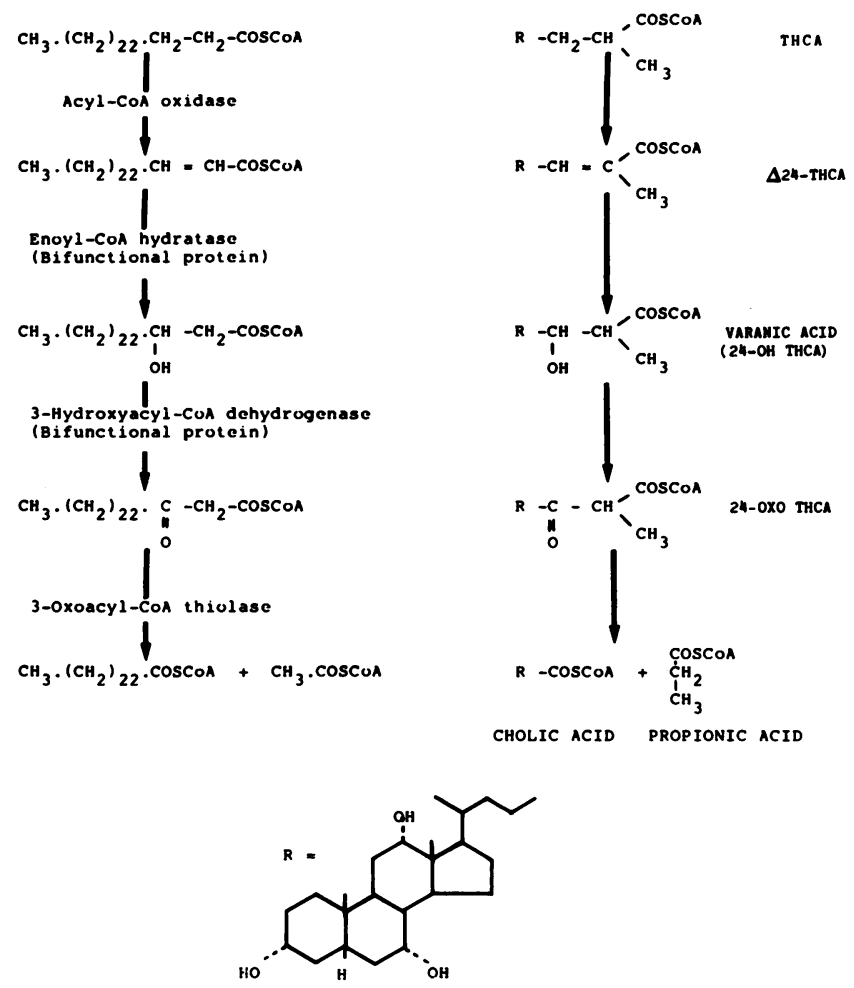

Figure 1. Peroxisomal pathway for $\beta$-oxidation of very long chain fatty acids and postulated pathway for oxidation of trihydroxycoprostanic acid (THCA) to cholic acid. Both pathways are blocked in the patient with peroxisomal 3-oxoacyl-CoA thiolase deficiency, providing evidence that the thiolase catalyzes the last step in both pathways.

pointed out that the accumulation of THCA in the patient's duodenal juice indicated that peroxisomal 3-oxoacyl-CoA thiolase participates in the oxidative cleavage of the THCA side chain. Since the thiolase catalyzes the last step in the reaction sequence proposed in Fig. 1, it would be reasonable to expect accumulation of the other intermediates in the pathway, $\triangle 24-T H C A$, varanic acid, and 24-oxo-THCA. The present study was undertaken firstly to test whether such intermediates could be detected, thereby providing further evidence for the occurrence of the proposed pathway in man, and secondly to provide reference bile acid profiles for comparison with profiles from patients suspected of having thiolase deficiency. A recent report documented three siblings with "pseudo-Zellweger" syndrome whose bile contained more varanic acid than THCA. Immunoreactive peroxisomal thiolase and bifunctional protein were present in the liver. It was suggested that these children may have a functional defect of the thiolase or the 3-hydroxyacyl-CoA dehydrogenase component of the bifunctional protein (10).

\section{Methods}

Subjects. The patient with CRM -ve peroxisomal 3-oxoacyl-CoA thiolase deficiency (L.C.) was described in reference 8. (The experiments that proved that she lacked immunoreactive peroxisomal thiolase protein are documented in reference 9.) Samples of duodenal juice (obtained when L.C. was aged $10 \mathrm{mo}$ ), plasma $\left(8 \frac{1}{2} \mathrm{mo}\right)$, and urine (5 d) had been stored at $-20^{\circ} \mathrm{C}$. The two siblings who had defective peroxisomal $\beta$-oxidation but whose precise enzyme defect has not been de- fined (N.B. and I.B.) were described in reference 10. Peroxisomes were present in the liver, reduced VLCFA oxidation was demonstrated in cultured skin fibroblasts and all three peroxisomal $\beta$-oxidation proteins were found using the immunoblotting technique. Duodenal juice, plasma and urine were collected from the children in this family when they were aged 8 mo (N.B.) and 2 wk (I.B.), respectively.

Six patients with Zellweger syndrome were compared with L.C., N.B., and I.B. They were aged between $7 \mathrm{~d}$ and 11 mo when studied. All were shown to have undetectable peroxisomes in their liver biopsy, reduced activity of dihydroxyacetone phosphate acyl transferase in skin fibroblasts and elevated plasma VLCFA and C27 bile acid concentrations $(11,12)$.

Procedures. Bile acids were extracted from urine by passing it through a cartridge of octadecylsilane bonded silica (11). Duodenal juice was diluted with $5 \mathrm{vol}$ of water and subjected to the same extraction procedure. The plasma was diluted with $4 \mathrm{vol}$ of $0.1 \mathrm{M}$ sodium hydroxide before passage through the cartridge (13). After washing with water and hexane, the bile acids from all three samples were eluted from their respective cartridges with $10 \mathrm{ml}$ of methanol. A small aliquot of the urine and bile extracts was analyzed by fast atom bombardment mass spectrometry [FAB-MS], recording negative ions between $\mathrm{m} / \mathrm{z} 200$ and 1,000 (11). All three extracts (bile, plasma, and urine) were analyzed by gas chromatography-mass spectrometry (GCMS) and two different methods were used for the hydrolysis of glycineand taurine-conjugated bile acids. For enzyme hydrolysis the extract was dissolved in $5 \mathrm{ml}$ of $0.15 \mathrm{M}$ sodium acetate buffer, $\mathrm{pH} 5.6$, and incubated with $90 \mathrm{U}$ of cholylglycine hydrolase for $3 \mathrm{~h}$ at $37^{\circ} \mathrm{C}(12,14$, 15). For alkaline hydrolysis the extract was dissolved in $15 \mathrm{ml}$ of $4.5 \mathrm{M}$ sodium hydroxide and heated to $130^{\circ} \mathrm{C}$ for $24 \mathrm{~h}$ in a Teflon ${ }^{\circ}$-lined stainless steel digestion vessel $(11,16)$. In the case of the plasma extract, the free bile acids were extracted on XAD-2 resin $(12,14,15)$; in the case of the bile and urine extracts, the bile acids were extracted on a Lipidex 1000 column after adjusting the $\mathrm{pH}$ to $3-4(11,16,17)$. Methylation with diazomethane, purification of methyl esters on a small column of neutral alumina and silylation (TriSil ${ }^{\circ}, 65^{\circ} \mathrm{C}, 1 \mathrm{~h}$ ) were performed as described previously $(11,12,14,15)$. However, the bile acid methyl ester trimethylsilyl ethers were then injected into the gas chromatograph in $\mathrm{TriSil}^{\otimes}$ without further purification on Lipidex 5000. In addition to the GC and GC-MS conditions described previously $(11,12)$, samples were analyzed on a Pye 4550 gas chromatograph fitted with a $25-\mathrm{m}, 0.25 \mathrm{~mm}$ i.d., fused silica capillary column coated with a chemically bonded BPA1 (OV1 equivalent) stationary phase ( $0.25 \mu \mathrm{m}$ film thickness) from Philips Scientific Co., Cambridge, UK. The bile acid derivatives were transferred to the column using an all-glass falling needle solid injection device with the injection point and initial column temperature at $200^{\circ} \mathrm{C}$. The carrier gas was helium $(2 \mathrm{ml} / \mathrm{min})$ and chromatograms were recorded from a flame ionization detector. Some of the GC-MS data was obtained using a Hewlett Packard 5970 mass selective detector (Hewlett Packard Co., Palo Alto, CA) and ChemStation data system. GC conditions were as described previously (12); the ion source temperature was $170^{\circ} \mathrm{C}$ and the ionization energy $70 \mathrm{eV}$. Spectra were compared with reference compounds (see below). For quantitation the chromatograms obtained with the flame ionization detector were used and the calculations were based on peak heights. For bile and urine a percent composition was determined assuming identical response factors for all bile acids. For plasma, concentrations in micromoles per liter were calculated by relating peak heights to that of the internal standard (14).

Reference compounds. Bile acids in the patients' samples were identified by comparing retention times and electron impact mass spectra with those of reference compounds. A number of useful reference compounds were obtained from the bile of Varanus salvator. The bile acids released by alkaline hydrolysis were methylated and the methyl esters separated by chromatography on a 2-g column of neutral alumina as described previously $(15,18)$. Table I lists some of the compounds obtained in this way. To obtain a single isomer of varanic acid, (24R,25S)-3 $\alpha, 7 \alpha, 12 \alpha, 24$-tetrahydroxy-5 $\beta$-cholestanoic acid, bile from Bombina orientalis was analyzed. Une et al. have shown that this 
Table I. Reference Bile Acids from the Bile of Varanus salvator: Chromatographic Behavior of Methyl Esters on a Neutral Alumina Column and GC-MS Characteristics of Methyl Ester TMS Ethers (Retention Indices, Major Ions in Electron Impact Mass Spectra, and Additional Evidence for Identities)

\begin{tabular}{|c|c|c|c|c|c|c|}
\hline \multirow[b]{2}{*}{ Bile acid methyl ester } & \multicolumn{3}{|c|}{$\begin{array}{l}\text { Eluted from } 2 \mathrm{~g} \text { alumina } \\
\text { column* by } 4 \mathrm{ml} \text { of:- }\end{array}$} & \multirow[b]{2}{*}{$\begin{array}{l}\text { Retention index } \\
\text { of TMS ether }\end{array}$} & \multirow[b]{2}{*}{ Major ions in mass spectrum of TMS ether } & \multirow[b]{2}{*}{$\begin{array}{l}\text { Additional evidence for } \\
\text { identity of TMS ether }\end{array}$} \\
\hline & Benzene & $\begin{array}{l}\text { Ethyl } \\
\text { acetate }\end{array}$ & Methanol & & & \\
\hline (24E)- $\triangle 24-\mathrm{THCA}$ & $30 \%$ & $70 \%$ & & 3,478 & $\begin{array}{l}663,588,573,498,483,408,393,343 \\
301,281,253,251 .\end{array}$ & $R_{R} T^{\delta}=$ quoted value (19) \\
\hline$\Delta 23$-THCA (isomer 1) & $30 \%$ & $70 \%$ & & $3,386)$ & $663,588,498,481,461,408,371, \underline{281}$ & $\mathbf{R R T}^{\S}=$ quoted value $(20)$ \\
\hline (isomer 2) & & $90 \%$ & $10 \%$ & $3,426)$ & 253. & Published spectrum (21) \\
\hline (isomer 3) & & $90 \%$ & $10 \%$ & $3,463)$ & & \\
\hline (24Z)- $\triangle 24-\mathrm{THCA}$ & & $90 \%$ & $10 \%$ & 3,520 & $\begin{array}{c}663,588,573,498,483,408,393,343 \\
\quad 301,281,253,251^{11}\end{array}$ & $\begin{array}{l}\mathbf{R R T}^{\delta}=\text { quoted value }(19) \\
\text { See also reference } 10\end{array}$ \\
\hline 24-Hydroxy-THCA-1 & & $80 \%$ & $20 \%$ & 3,600 & $753,588,498,408,343,321,281, \underline{253}{ }^{\prime \prime}$ & $\begin{array}{l}\text { Identical with major bile acid } \\
\text { of Bombina orientalis } \\
(24 \mathrm{R}, 25 \mathrm{~S})\end{array}$ \\
\hline 24-Hydroxy-THCA-2 & & $80 \%$ & $20 \%$ & 3,612 & $753,588,498,408,343,321,281, \underline{253}^{\prime \prime}$ & \\
\hline
\end{tabular}

* Neutral alumina activity grade $\mathrm{V}(15,18) .{ }^{\ddagger}$ Base peak underlined. ${ }^{\S}$ Relative retention time on OV 17 packed column. " Full spectrum in reference 22.

is the only isomer present in Bombina bile and that it is found here in unconjugated form, obviating the need for alkaline hydrolysis (23). Bile acids from Bombina orientalis were extracted on Lipidex 1000 and analyzed by GC-MS as described above. The major peak had a retention index (3600) and mass spectrum identical to those of "24-hydroxy-THCA-1" from Varanus bile. (12) Thus 24-hydroxyTHCA-1 was indistinguishable on capillary GC-MS from (24R,25S)$3 \alpha, 7 \alpha, 12 \alpha, 24$-tetrahydroxy-5$\beta$-cholestanoic acid; 24 -hydroxy-THCA2 from Varanus bile was definitely not the $(24 R, 25 S)$ isomer.

\section{Results}

Duodenal juice. The capillary GC chromatogram obtained when duodenal bile from L.C. was analyzed after enzyme hydrolysis is shown in Fig. 2. The major bile acids were chenodeoxycholic acid (peak 1) and 24-hydroxy-THCA-1 (peak 11). Next in order of magnitude were 24-hydroxy-THCA-2 (peak 12), THCA (peak 6), and cholic acid (peak 2).

Among the smaller peaks a series of unsaturated analogues of THCA were identified. Peak 8 was (24E)- $\Delta 24-$ THCA and peak 10 was the (24Z)- isomer. Peaks 5 and 7 were incompletely resolved from THCA but were clearly identical with two of the isomers of $\triangle 23$-THCA present in Varanus bile (the isomers with RI values of 3426 and 3463). By comparison with varanic acid, other side-chain hydroxylated derivatives of THCA were present in very modest amounts, peaks 13 and 14

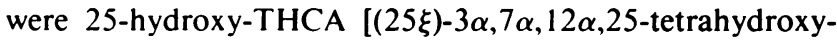

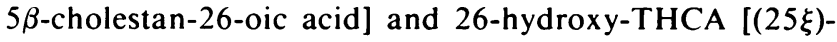
$3 \alpha, 7 \alpha, 12 \alpha, 26$-tetrahydroxy-cholestan-27-oic acid], respectively.

Peak $4(\mathrm{RI}=3320)$ produced a spectrum containing the following ions: $\mathrm{m} / \mathrm{z} 456,366,351,343,294,283,281,253$. The spectrum was very similar to that quoted by Noma et al. for $3 \alpha, 7 \alpha, 12 \alpha$-trihydroxy-27-nor-5 $\beta$-cholestan-24-one (24). The retention time was also in agreement: when the derivatized sample from L.C. was analyzed on a packed OV 1 column peak 4 had a relative retention time of 1.21 using as a reference peak the methyl ester TMS ether of cholic acid; the figure quoted by Noma et al. for synthetic $3 \alpha, 7 \alpha, 12 \alpha$-trihydroxy-27nor-5 $\beta$-cholestan-24-one is 1.20 .

GC-MS analysis of enzyme-hydrolyzed bile from patients with Zellweger syndrome (N.W. and A.P. in reference 12) re-

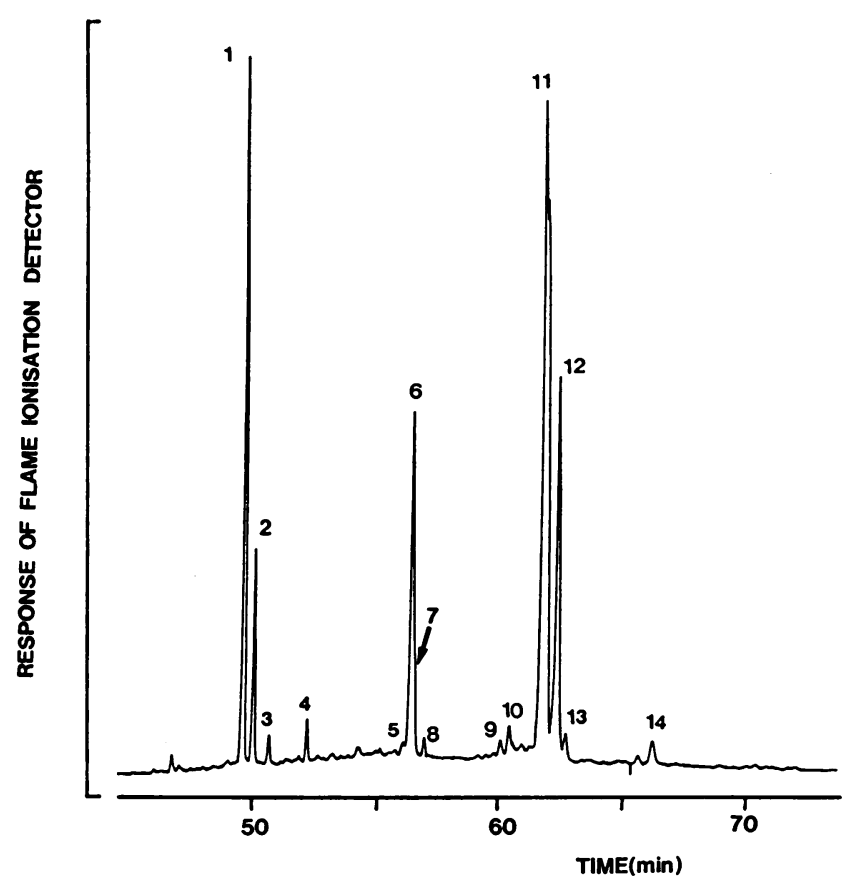

Figure 2. Capillary gas chromatogram of the bile acid methyl ester trimethylsilyl ethers prepared from the duodenal juice of patient L.C. (CRM-ve peroxisomal thiolase deficiency). Conjugated bile acids were hydrolysed with cholylglycine hydrolase. Identities indicated by mass spectrometry were: $I$, chenodeoxycholic acid; 2 , cholic acid; 3 , ursodeoxycholic acid ( $3 \alpha, 7 \beta$-dihydroxy-5 $\beta$-cholanic acid); 4 $3 \alpha, 7 \alpha, 12 \alpha$-trihydroxy-27-nor-5 $\beta$-cholestan-24-one; 5, $\Delta 23$-THCA; 6 , THCA; 7, $\triangle 23$-THCA; 8, (24E)- $\triangle 24-\mathrm{THCA}$; , unknown; 10, (24Z)224-THCA; 11,24 -hydroxy-THCA-1; 12, 24-hydroxy-THCA-2; 13 , 25-hydroxy-THCA; 14, 26-hydroxy-THCA. 
vealed that these patients secreted bile with a much lower percentage of varanic acid (Table II). The percentage of the C24hydroxylated derivatives of THCA was similar to the percentages of C25- and C26-hydroxylated derivatives of THCA in the bile of these patients. By contrast, enzyme hydrolysis of bile from I.B. and N.B., the two siblings with suspected thiolase or 3-hydroxyacyl-CoA dehydrogenase deficiency (10) yielded a similar mixture of bile acids to that found in the bile from L.C. (Table II). In particular, varanic acid was the major C27 bile acid and both $\triangle 24-T H C A$ [the (24E)- and (24Z)isomers] and $\triangle 23-T H C A$ (two isomers) were detected. There were, however, some differences: Firstly, the varanic acid in the duodenal juice from I.B. and N.B. was almost exclusively the 24-hydroxy-THCA-1. Secondly, a small peak of retention index 3593 was present in enzyme-hydrolyzed bile from both N.B. and I.B. but not L.C. This peak produced a spectrum containing ions $\mathrm{m} / \mathrm{z} 604,514$ and 424 and we previously suggested that it may be 24-oxo-THCA (10). However, an alternative explanation for these ions would be the presence of a tetrahydroxycholestenoic acid with one hydroxyl group not derivatised. Similar tetrahydroxycholestenoic acids were found in the urine of N.B. (see below). The bile alcohol detected in bile from L.C. $(3 \alpha, 7 \alpha, 12 \alpha$-trihydroxy-27-nor-5 $\beta$ cholestan-24-one) was also found in the bile from N.B. and I.B.

Substantial amounts of $\mathrm{C} 27$ bile acids were detected when the bile samples were analyzed following enzyme hydrolysis of conjugates. However, the FAB-MS analysis indicated that these were probably actually present largely in unconjugated form. The negative ion FAB mass spectrum obtained from the duodenal bile of L.C. is shown in Fig. 3. The major ions are listed in Table III alongside the major ions in the FAB mass spectra from the bile of I.B. and N.B. and the proposed identitie: of the ions. All three FAB mass spectra showed a major peak $(\mathrm{m} / \mathrm{z} 465)$ attributable to unconjugated varanic acid. Only in the bile from I.B. was a peak attributable to tauro-varanic acid (m/z 572) detected. By contrast, all three analyses revealed that $\mathrm{C} 24$ bile acids were present as the glycine and

Table II. Percentage Composition of the Bile Acid Mixture Obtained from the Bile of Patients with Absent Peroxisomes, with CRM-ve Thiolase Deficiency and with an Unknown Defect of Peroxisomal $\beta$-oxidation

\begin{tabular}{|c|c|c|c|c|c|}
\hline \multirow[b]{2}{*}{ Bile acid } & \multicolumn{2}{|c|}{$\begin{array}{c}\text { Absent } \\
\text { peroxisomes }\end{array}$} & \multirow{2}{*}{$\begin{array}{c}\begin{array}{c}\text { Thiolase } \\
\text { deficiency }\end{array} \\
\text { LC }\end{array}$} & \multicolumn{2}{|c|}{$\begin{array}{l}\text { Unknown defect of } \\
\text { peroxisomal } \beta \text {-oxidation }\end{array}$} \\
\hline & NW & AP & & IB & $\mathrm{NB}^{*}$ \\
\hline Chenodeoxycholic & 11.3 & 8.5 & $28.6(15.5)$ & $3.1(6.7)$ & $21(10.4)$ \\
\hline Cholic & 73.8 & 45.2 & $8.5(11.6)$ & $51.2(18.6)$ & $24(19.6)$ \\
\hline THCA & 4.3 & 17.2 & $13.8(9.5)$ & $8.6(40.1)$ & $10(6.0)$ \\
\hline (24E)- $\triangle 24-T H C A$ & $<0.2$ & 0.2 & $0.7(0.9)$ & $0.3(2.0)$ & $2(0.7)$ \\
\hline$(24 Z)-\Delta 24-T H C A$ & $<0.2$ & $<0.3$ & $1.0(7.2)$ & $0.4(20.2)$ & $13(9.1)$ \\
\hline 24-Hydroxy-THCA-1 & 0.8 & 3.4 & $26.5(21.5)$ & $21.7(<1.0)$ & $27(13.6)$ \\
\hline 24-Hydroxy-THCA-2 & 2.4 & 5.1 & $15.5(20.5)$ & $<0.5(<1.0)$ & $2(11.1)$ \\
\hline 25-Hydroxy-THCA & 0.6 & 3.3 & $1.0(2.4)$ & $1.8(1.2)$ & $<2(2.3)$ \\
\hline 26-Hydroxy-THCA & 2.9 & 5.4 & $0.8(1.0)$ & $2.1(0.6)$ & $<2(3.2)$ \\
\hline
\end{tabular}

The figures have been computed from the chromatograms obtained after enzyme hydrolysis except those in brackets that relate to alkali hydrolyzed bile. * Only a small amount of sample was available for analysis of this sample following enzyme hydrolysis and therefore only the major components could be identified.

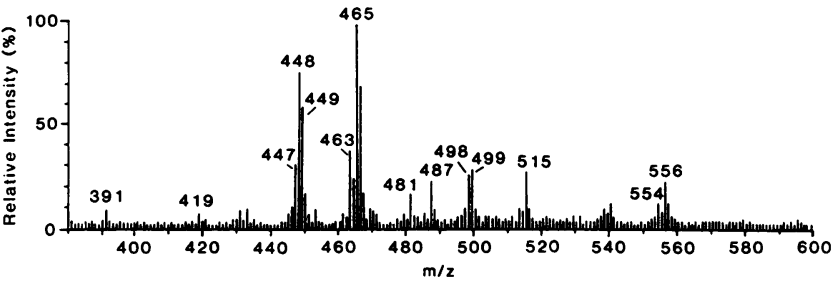

Figure 3. Negative ion fast atom bombardment mass spectrum of a crude extract of bile acids from the duodenal juice of patient L.C. with CRM -ve peroxisomal thiolase deficiency. The postulated origins of the major ions are indicated in Table III.

taurine conjugates. Peaks that could have arisen from unconjugated THCA (m/z 449) and from $\Delta 24$-THCA and $\Delta 23$ THCA ( $\mathrm{m} / \mathrm{z} 447)$ were readily detectable above background in the FAB mass spectra from L.C. and N.B. but in the bile from I.B. these compounds appeared to be present only as taurine conjugates (m/z 556 and 554 , respectively). The fact that they were detected on the GC-MS analysis indicates that some enzyme hydrolysis of the taurine conjugates must have occurred. This was confirmed when the FAB-MS analysis of bile from L.C. was repeated after enzymatic deconjugation. As expected the glycocholate peak ( $\mathrm{m} / \mathrm{z} 464)$ disappeared and a cholic acid

Table III. Proposed Origins and Relative Intensities of Ions in the Negative Ion FAB Mass Spectra of Duodenal Juice from LC (CRM-ve Peroxisomal Thiolase Deficiency) and I.B. and N.B. (Siblings with an Unknown Defect of Peroxisomal $\beta$-oxidation)

\begin{tabular}{|c|c|c|c|c|}
\hline \multirow[b]{2}{*}{ Bile acid/alcohol } & \multirow{2}{*}{$\begin{array}{l}\text { Mass charge ratio } \\
\text { of }[\mathrm{M}-\mathrm{H}]^{-} \text {ion }\end{array}$} & \multicolumn{3}{|c|}{$\begin{array}{l}\text { \% Relative intensity } \\
\text { in FAB mass } \\
\text { spectrum of bile }\end{array}$} \\
\hline & & LC & IB & NB \\
\hline Varanic & 465 & 100 & 96 & 100 \\
\hline Sodium salt & 487 & 25 & & \\
\hline Taurine conjugate & 572 & & 65 & \\
\hline Chenodeoxycholic & 391 & 8 & & \\
\hline Glycine conjugate & 448 & 78 & & 35 \\
\hline Taurine conjugate & 498 & 28 & 38 & 33 \\
\hline Trihydroxycoprostanic (THCA) & 449 & 60 & & 21 \\
\hline Taurine conjugate & 556 & 23 & 70 & 29 \\
\hline Cholic & 407 & & & \\
\hline Glycine conjugate & 464 & 27 & & 39 \\
\hline Taurine conjugate & 514 & & 100 & 23 \\
\hline$\Delta 24-\mathrm{THCA}$ and $\Delta 23$-THCA & 447 & 32 & & 25 \\
\hline Taurine conjugate & 554 & 14 & 55 & 23 \\
\hline \multicolumn{5}{|l|}{$3 \alpha, 7 \alpha, 12 \alpha$-trihydroxy-27-nor- } \\
\hline $5 \beta$-cholestan-24-one & 419 & 8 & & \\
\hline Sulfate & 499 & 30 & 28 & 25 \\
\hline \multicolumn{5}{|l|}{ ?Tetrahydroxy-27-nor-5 $\beta$ - } \\
\hline cholestan-24-one & 435 & & & \\
\hline Sulfate & 515 & 30 & & 35 \\
\hline \multicolumn{5}{|l|}{$\begin{array}{l}\text { ?Trihydroxy-oxo-cholestanoic or } \\
\text { tetrahydroxy-cholestenoic }\end{array}$} \\
\hline acid & 463 & 39 & 65 & 38 \\
\hline Taurine conjugate & 570 & & 40 & \\
\hline ?Pentahydroxy-cholestanoic acid & 481 & 18 & 43 & 17 \\
\hline
\end{tabular}


peak $(\mathrm{m} / \mathrm{z} 407)$ appeared, the glycochenodeoxycholate and taurochenodeoxycholate peaks (m/z 448 and 498) disappeared and the chenodeoxycholate peak $(\mathrm{m} / \mathrm{z} 391)$ became larger. In addition, however, the peaks $\mathrm{m} / \mathrm{z} 556$ and 554 disappeared indicating that there had been appreciable enzyme hydrolysis of taurine-conjugated THCA and $\Delta$-THCA.

The FAB-MS analysis of bile from L.C. indicated a small peak of $\mathrm{m} / \mathrm{z}$ ratio 419 . This ion was attributed to the presence of the bile alcohol, $3 \alpha, 7 \alpha, 12 \alpha$-trihydroxy-27-nor- $5 \beta$-cholestan-24-one (in nonsulfated form) in bile from L.C. and suggested that it did not arise by decarboxylation of 24-oxoTHCA during the sample work-up. The presence of a much larger ion of $\mathrm{m} / \mathrm{z}$ ratio 499 may indicate that the $\mathrm{C} 26$ bile alcohol was also present as the sulfate (although the same ion would also be produced by a sulfated trihydroxy $\mathrm{C} 27$ bile alcohol). The GC-MS analysis as performed provided no information viz a viz the structures of sulfated bile alcohols. Whereas nonsulfated bile alcohols are extracted from aqueous solutions by Lipidex 1000, the sulfates are not extracted. In any case, no solvolysis step was included in the sample work-up.

Evidence was sought for the presence of 24-oxo-THCA in the bile from L.C. This should have produced an ion of $\mathrm{m} / \mathrm{z}$ ratio 463 on FAB-MS analysis. Such an ion was present in the spectrum from L.C.; its intensity was $40 \%$ that of $\mathrm{m} / \mathrm{z} 465$, the $[\mathrm{M}-\mathrm{H}]^{-}$ion of varanic acid. This is perhaps more intense than one would expect for a [M-H-2 $]^{-}$ion arising by fragmentation of varanic acid and does suggest the presence of either 24-oxoTHCA or a tetrahydroxy-cholestenoic acid. However, no corresponding compound was seen on the GC-MS analysis and therefore we do not have conclusive evidence that 24-oxoTHCA had accumulated in the bile as a consequence of peroxisomal thiolase deficiency.

When GC-MS analyses of bile were performed after alkaline hydrolysis, the results suggested that artefacts were produced using this method. For example enzyme-hydrolyzed bile from I.B. showed a large peak attributable to (24R,25S)varanic acid. On the chromatogram obtained following alkaline hydrolysis this peak was absent and a large peak due to (24Z)- $\Delta 24-T H C A$ was present instead. This indicated that alkali-induced dehydration of 24-hydroxy-THCA-1 to (24Z)$\triangle 24-T H C A$ can occur. Alkali-hydrolyzed bile from L.C. also contained a higher percentage of (24Z)- $\Delta 24-T H C A$ than the enzyme-hydrolyzed samples (Table II). Enzyme-hydrolyzed bile from L.C. contained mostly the 24-hydroxy-THCA-1 but the alkali-hydrolyzed sample contained an equal amount of the 24-hydroxy-THCA-2 indicating that alkaline hydrolysis had converted some of the 24-hydroxy-THCA-1 to 24-hydroxy-THCA-2.

Plasma. C27 bile acids were detected on GC-MS analysis of the plasma of patient L.C. with CRM -ve thiolase deficiency (enzyme hydrolyzed sample). The concentrations were considerably lower than the concentrations of chenodeoxycholic and cholic acids (Table IV). The concentrations of THCA and varanic acid were approximately equal and no C29-dicarboxylic acid $(3 \alpha, 7 \alpha, 12 \alpha$-trihydroxy-27a,27b-dihomo-5 $\beta$-cholestan-26,27b-dioic acid) was detected. This plasma bile acid pattern was also observed in the two siblings with the unknown defect of peroxisomal $\beta$-oxidation except that in these cases the concentrations of THCA and of 24-hydroxy-THCA-1 were greater, similar to those of chenodeoxycholic and cholic acid. By contrast, in the plasma of six infants with undetectable peroxisomes, the concentration of THCA was always greater than that of varanic acid and the C-29 dicarboxylic acid was always readily detectable.

Urine. After alkaline hydrolysis, GC-MS analysis of urine from L.C. showed that all bile acids were present at low concentration (as in the plasma). THCA, $\triangle 24-T H C A$, and varanic acid were all present but again, the concentrations were less than that of cholic acid. The major components in the mixture of $\mathrm{C} 24$ and $\mathrm{C} 27$ bile acids in the urine were as follows: cholic acid, $38 \%$; THCA，8.2\%; 24-hydroxy-THCA-1, $6.6 \%$; $3 \beta, 7 \alpha, 12 \alpha$-trihydroxy-5 $\beta$-cholanic acid, $6.6 \% ; 24$-hydroxyTHCA-2, 4.1\%; (24E)- $\triangle 24-\mathrm{THCA}, 3.9 \%$; (24Z)- $\Delta 24-\mathrm{THCA}$, $3.8 \%$; chenodeoxycholic acid, $3.1 \%$. The results of GC-MS analyses of urine from our patients with undetectable peroxisomes have been described previously (11). Tetrahydroxycholestanoic acids predominated but among these varanic acid was a minor component; the major tetrahydroxycholestanoic acids were nuclear-hydroxylated derivatives of THCA, e.g., $1 \beta$-hydroxy-THCA, $6 \alpha$-hydroxy-THCA. Urine from N.B., when analyzed by GC-MS after alkaline hydrolysis contained the following major components: $1 \beta, 3 \alpha, 7 \alpha, 12 \alpha$-tetrahydroxy$5 \beta$-cholanic acid, $16 \% ; 1 \beta, 3 \alpha, 7 \alpha, 12 \alpha$-tetrahydroxy- $5 \beta$-cholestanoic acid plus $3 \alpha, 6 \alpha, 7 \alpha, 12 \alpha$-tetrahydroxy-5 $\beta$-cholestanoic acid, 12\%; (?3,4,7,12-)tetrahydroxycholestenoic acid (RI = 3669), 12\%; cholic acid, 10\%; THCA, 8\%; tetrahydroxycholestenoic acid $(\mathrm{RI}=3632), 8 \%$. The tentatively identified tetrahydroxycholestenoic with a retention index of 3669 produced a mass spectrum containing the following ions: 766(M), 751(M-15), $\underline{586}(\mathrm{M}-[2 \times 90]), 496(\mathrm{M}-[3 \times 90]), 406(\mathrm{M}-[4$ $\times 90]), 331(\mathrm{C} 3-\mathrm{C} 7$ with three TMS substituents), 259, 253, 251. The second major tetrahydroxycholestenoic acid in the urine of N.B. (RI 3632) produced a spectrum with the following ions:- 676(M-90), 646, 586(M-[2 × 90]), 556, 496(M-[3 $\times 90]), 406(\mathrm{M}-[4 \times 90]), 331,281,253,251$, 201. Further work is required to identify these compounds fully. The tetrahydroxycholestenoic acids in the urine were present as taurine conjugates producing an ion at $\mathrm{m} / \mathrm{z} 570$ in the FAB-MS analy-

Table IV. Plasma Bile Acid Concentrations in L.C. (CRM-ve Peroxisomal Thiolase Deficiency), I.B. and N.B. (Unknown Defect of Peroxisomal $\beta$-oxidation) and Six Infants with Absent Peroxisomes (Zellweger Syndrome)

\begin{tabular}{|c|c|c|c|c|}
\hline \multirow[b]{3}{*}{ Bile acid } & \multicolumn{4}{|c|}{ Plasma concentration ( $\mu \mathrm{mol} / \mathrm{liter})$} \\
\hline & \multirow{2}{*}{$\begin{array}{c}\begin{array}{c}\text { Thiolase } \\
\text { deficiency }\end{array} \\
\text { L.C. }\end{array}$} & \multicolumn{2}{|c|}{$\begin{array}{l}\text { Unknown defect } \\
\text { of peroxisomal } \\
\beta \text {-oxidation }\end{array}$} & \multirow{2}{*}{$\begin{array}{c}\begin{array}{c}\text { Absent } \\
\text { peroxisomes } \\
(n=6)\end{array} \\
\text { Mean (Range) }\end{array}$} \\
\hline & & I.B. & N.B. & \\
\hline Chenodeoxycholic & 1.1 & 2.0 & 1.4 & $6.5(0.5-25)$ \\
\hline Cholic & 2.4 & 4.2 & 2.7 & $7.0(1.1-29)$ \\
\hline THCA & 0.14 & 1.7 & 8.9 & $12.1(2.9-30)$ \\
\hline (24E)- $\Delta 24-\mathrm{THCA}$ & 0.10 & 0.06 & $<0.05$ & $0.04(0-0.08)$ \\
\hline (24Z)- $\triangle 24-\mathrm{THCA}$ & 0.07 & 0.3 & 1.1 & $0.02(0-0.05)$ \\
\hline 24-Hydroxy-THCA-1 & 0.08 & 4.4 & 3.9 & $0.92(0.6-2.3)$ \\
\hline 24-Hydroxy-THCA-2 & $<0.01$ & 0.2 & $<0.05$ & $1.0(0.6-4.6)$ \\
\hline 25-Hydroxy-THCA & $<0.01$ & 0.4 & 0.7 & $1.1(0-4.0)$ \\
\hline 26-Hydroxy-THCA & 0.09 & 0.9 & 0.4 & $2.1(0.8-4.3)$ \\
\hline C29-Dicarboxylic* & $<0.01$ & $<0.01$ & $<0.01$ & $12.3(3.4-29)$ \\
\hline
\end{tabular}

* $3 \alpha, 7 \alpha, 12 \alpha$-Trihydroxy-27a,27b-dihomo- $5 \beta$-cholestan-26,27b-dioic acid. 
sis. This, together with an ion at $\mathrm{m} / \mathrm{z} 572$ from the taurineconjugated tetrahydroxycholestanoic acids, were the dominant bile acid ions in the urine spectrum.

FAB-MS analysis of urine from L.C. (CRM -ve peroxisomal thiolase deficiency) did not show an ion at $\mathrm{m} / \mathrm{z} 572$ or an ion at $\mathrm{m} / \mathrm{z} 570$. A small ion of $\mathrm{m} / \mathrm{z} 556$ just above background was tentatively attributed to the presence of taurine-conjugated THCA. When the results of the GC-MS analysis were available (see above) it became clear that the low intensity of ions attributable to $\mathrm{C} 27$ bile acids in the FAB mass spectrum from the urine of L.C. was attributable to the small amounts of C27 bile acids present.

\section{Discussion}

The results reported above have important implications with regard to proposed pathways for bile acid and alcohol synthesis in man, the nature of the enzyme defect in N.B. and I.B. and the use of bile, plasma, and urine analyses to detect patients with peroxisomal thiolase deficiency.

Pathways of bile acid and bile alcohol synthesis. The accumulation of large amounts of varanic acid in bile in peroxisomal thiolase deficiency provides strong evidence for the pathway in Fig. 1. The presence of $\Delta 24-\mathrm{THCA}$ also supports this pathway. Conclusive evidence for the presence of 24-oxoTHCA was not obtained and must await improvements in methodology. Evidence was obtained to indicate that the stereochemistry of the varanic acid produced during cholic acid synthesis in man is probably the same as in the rat. Une et al. have shown that when either (25R)- or (25S)-THCA is incubated with rat liver homogenates it is (24E)- $\Delta 24-\mathrm{THCA}$ and $(24 R, 25 S)$-varanic acid that are produced (25). In the patient with peroxisomal thiolase deficiency $(24 \mathrm{R}, 25 \mathrm{~S})$-varanic acid was probably the major isomer that accumulated and (24E)$\Delta 24-T H C A$ was certainly present.

If a single isomer of varanic acid is produced during conversion of THCA to cholic acid in man, how can we explain the presence of the second isomer in the bile of L.C.? The answer may lie in the fact that peroxisomes contain an epimerase capable of changing the chirality at C3 of 3-hydroxyacylCoA (26); if active on the accumulating (24R,25S)-varanic acid in L.C., this enzyme would convert (24R,25S)-varanic acid to (24S,25S)-varanic acid. Alternatively, (24S,25S)-varanic acid might arise from the action of a $D$-3-hydroxyacid dehydrogenase on 24-oxo-THCA.

$\Delta 23-$ THCA is not on the pathway shown in Fig. 1 and so its presence in the bile of L.C. also requires explanation. Again the answer may lie in the operation of a peroxisomal isomerase, this time the $\Delta 3$-,trans- $\Delta 2$-enoyl-CoA isomerase (27). This enzyme, if active on (24E)- $\Delta 24-\mathrm{THCA}$ would lead to the formation of $\triangle 23$-THCA.

The detection of $3 \alpha, 7 \alpha, 12 \alpha$-trihydroxy-27-nor-5 $\beta$-choles$\tan$-24-one in the bile of L.C. is of interest with regard to the postulated mechanism for synthesis of $\mathrm{C} 26$ bile alcohols in man. When Noma et al. found this oxo bile alcohol in the bile of Rana catesbeiana, it led them to postulate a pathway involving decarboxylation of 24-oxo-THCA as the mechanism by which $5 \beta$-ranol (27-nor-5 $\beta$-cholestan- $3 \alpha, 7 \alpha, 12 \alpha, 24,26-$ pentol) is synthesized from cholesterol, $5 \beta$-cholestan$3 \alpha, 7 \alpha, 12 \alpha$-triol, and varanic acid in this amphibian (24). Karlaganis et al. postulated that the same decarboxylation reaction in man could lead to the synthesis of $\mathrm{C} 26$ bile alcohols, e.g., 27-nor-5 $\beta$-cholestan-3 $\alpha, 7 \alpha, 12 \alpha, 24,25$-pentol (28). The fact that $3 \alpha, 7 \alpha, 12 \alpha$-trihydroxy-5 $\beta$-cholestan-24-one accumulates when metabolism of 24-oxo-THCA is blocked by peroxisomal thiolase deficiency suggests that this pathway may indeed occur in man.

The remaining compounds identified in this study can be accounted for on the basis of microsomal hydroxylation reactions. Microsomal hydroxylation of THCA in patients with Zellweger syndrome leads to the production of $1 \beta$ - and $6 \alpha$-hydroxy derivatives (11). It probably accounts for the small amounts of varanic acid in their bile and for the presence of 25-hydroxy-and 26-hydroxy-THCA in the bile of L.C. and the Zellweger patients.

Chenodeoxycholic acid was present at high concentration in bile from L.C., whereas intermediates in the $\beta$-oxidation of DHCA were not detected. This suggests that peroxisomal $\beta$ oxidation of DHCA may not be the only pathway for chenodeoxycholic acid synthesis. Alternatively, the absence of DHCA, $\Delta 24-\mathrm{DHCA}$, and 24-hydroxy-DHCA may be explained by the fact that DHCA can undergo $12 \alpha$-hydroxylation to produce THCA.

The synthesis of unconjugated varanic acid instead of conjugates of cholic acid does not appear to be associated with significant hepatic dysfunction. Standard liver function tests and the total bile acid concentration in the blood were normal in L.C. The concentration of unconjugated varanic acid in the plasma was lower than the concentrations of chenodeoxycholic and cholic acids, suggesting efficient clearance of varanic acid by the liver. The liver was histologically normal except for a mild degree of stellate portal fibrosis (8). Patients with absent peroxisomes by contrast often have frank cirrhosis.

The enzyme defect in N.B. and I.B. The accumulation of varanic acid in the bile of N.B. and I.B. indicates a defect either of the 3-hydroxyacyl-CoA dehydrogenase component of the bifunctional protein or of the thiolase. The presence of $3 \alpha, 7 \alpha, 12 \alpha$-trihydroxy-27-nor-5 $\beta$-cholestan-24-one argues against a defect in the enzyme that introduces the 24-oxo group and in favor of thiolase deficiency. However, N.B. and I.B. clearly differed from L.C. in that bile from N.B. and I.B. analyzed after enzyme deconjugation contained almost exclusively 24-hydroxy-THCA-1, whereas that from L.C. contained 24-hydroxy-THCA-2 in addition. If the second isomer of varanic acid in L.C. was derived from 24-oxo-THCA then this would suggest that N.B. and I.B. had 3-hydroxyacyl-CoA dehydrogenase deficiency. Preliminary results from Wanders et al. on fibroblasts from N.B. and I.B. also indicate deficiency of the 3-hydroxyacyl-CoA dehydrogenase activity of the bifunctional protein (29). Studies of CRM -ve bifunctional protein deficiency make it clear that this protein is involved in the metabolism of THCA as well as VLCFA (30).

Plasma and urine bile acid analyses in the diagnosis of peroxisomal thiolase deficiency. Screening for a peroxisomopathy by FAB-MS of urinary bile salts would have failed to detect an abnormality in L.C. Analysis of plasma bile acids by GC-MS would have detected the presence of traces of THCA and varanic acid but only if the results were being scrutinized carefully to detect such small amounts. Thus these methods are inferior to estimation of VLCFA in screening for the condition. However, the results of the examination of duodenal juice are so clear that this will probably remain an important supplementary test when a defect of peroxisomal $\beta$-oxidation is suspected in an infant. Simple noninvasive methods exist for obtaining the small amount of bile required to determine the 
bile acid profile and, using FAB-MS analysis, an answer can be obtained quickly.

It should be stressed that caution is necessary when interpreting FAB mass spectra. It is particularly difficult to be certain from FAB-MS alone that an unsaturated bile acid is present if the saturated analogue is also present in the sample. This is because for all bile acids a significant $[\mathrm{M}-\mathrm{H}-2]^{-}$ion accompanies the $[\mathrm{M}-\mathrm{H}]^{-}$ion and depending upon the ionization conditions this $[\mathrm{M}-\mathrm{H}-2]^{-}$ion may be $20-40 \%$ of the intensity of the parent ion. Also it is important to remember which body fluid is being studied. For example an ion of $\mathrm{m} / \mathrm{z} 465$ in urine is more likely to represent androsterone or etiocholanolone glucuronide than unconjugated varanic acid.

\section{Acknowledgments}

This work was supported by a grant from the Child Health Research Appeal Trust. GC-MS equipment was generously donated to the Institute of Child Health by Asprey \& Co. PLC, London, U.K.

\section{References}

1. Björkhem, I. 1984. Mechanism of bile acid biosynthesis in mammalian liver. In Sterols and Bile Acids. New Comprehensive Biochemistry. Vol. 12. H. Danielsson and J. Sjövall, editors. Elsevier/ North Holland Publishing Co., Amsterdam. 231-278.

2. Kase, B. F., I. Björkhem, and J. I. Pedersen. 1983. Formation of cholic acid from $3 \alpha, 7 \alpha, 12 \alpha$-trihydroxy- $5 \beta$-cholestanoic acid by rat liver peroxisomes. J. Lipid Res. 24:1560-1567.

3. Miura, S., M. Mori, M. Takiguchi, M. Tatibana, S. Furuta, S. Miyazawa, and T. Hashimoto. 1984. Biosynthesis and intracellular transport of enzymes of peroxisomal $\beta$-oxidation. J. Biol. Chem. 259:6397-6402.

4. Tager, J. M., W. A. Ten Harmsen Van Der Beek, R. J. A. Wanders, T. Hashimoto, H. S. A. Heymans, H. Van den Bosch, R. B. H. Schutgens, and A. Schram. 1985. Peroxisomal $\beta$-oxidation proteins in the Zellweger syndrome. Biochem. Biophys. Res. Commun. 126:1269-1275.

5. Swell, L., J. Gustafsson, H. Danielsson, C. C. Schwartz, and Z. R. Vlahcevic. 1981. Biosynthesis of bile acids in man. An in vivo evaluation of the conversion of $\mathrm{R}$ and $\mathrm{S} 3 \alpha, 7 \alpha, 12 \alpha$-trihydroxy-5 $\beta$ cholestanoic acid and $3 \alpha, 7 \alpha, 12 \alpha, 24 \xi$-tetrahydroxy-5 $\beta$-cholestanoic acids to cholic acid. J. Biol. Chem. 256:912-916.

6. Moser, H. W. 1988. The peroxisome: nervous system role of a previously underrated organelle. Neurology. 38:1617-1627.

7. Kase, B. F., I. Björkhem, P. Haga, and J. I. Pedersen. 1985. Defective peroxisomal cleavage of the $\mathrm{C}_{27}$-steroid side chain in the cerebro-hepato-renal syndrome of Zellweger. J. Clin. Invest. 75:427435.

8. Goldfischer, S., J. Collins, I. Rapin, P. Neumann, W. Neglia, A. Spiro, T. Ishii, F. Roels, J. Vamecq, and F. Van Hoof. 1986. PseudoZellweger syndrome: deficiencies in several peroxisomal oxidative activities. J. Pediatr. 108:25-32.

9. Schram, A. W., S. Goldfischer, C. W. T. van Roermund, E. M. Brouwer-Kelder, J. Collins, T. Hashimoto, H. S. A. Heymans, H. Van den Bosch, R. B. H. Schutgens, J. M. Tager, and R. J. A. Wanders. 1987. Human peroxisomal 3-oxoacyl-coenzyme A thiolase deficiency. Proc. Natl. Acad. Sci. USA. 84:2494-2496.

10. Clayton, P. T., B. D. Lake, M. Hjelm, J. B. P. Stephenson, G. T. N. Besley, R. J. A. Wanders, A. W. Schram, J. M. Tager, R. B. H. Schutgens and A. M. Lawson. 1988. Bile acid analyses in "pseudo-Zellweger" syndrome; clues to the defect in peroxisomal $\beta$ oxidation. J. Inher. Metab. Dis. 11(Suppl. 2):165-168.

11. Lawson, A. M., M. J. Madigan, D. Shortland, and P. T. Clayton. 1986. Rapid diagnosis of Zellweger syndrome and infantile Refsum's disease by fast atom bombardment-mass spectrometry of urine bile salts. Clin. Chim. Acta. 161:221-231.
12. Clayton, P. T., B. D. Lake, N. A. Hall, D. B. Shortland, R. A. Carruthers, and A. M. Lawson. 1987. Plasma bile acids in patients with peroxisomal dysfunction syndromes: Analysis by capillary gas chromatography-mass spectrometry. Eur. J. Pediatr. 146:166-173.

13. Setchell, K. D. R., and J. Worthington. 1982. A rapid method for the quantitative extraction of bile acids and their conjugates from serum using commercially available reverse-phase octadecyl bonded silica cartridges. Clin. Chim. Acta. 125:135-144.

14. Clayton, P. T., and D. P. R. Muller. 1980. A simplified gas liquid chromatographic method for the estimation of non-sulphated plasma bile acids. Clin. Chim. Acta. 105:401-405.

15. Clayton, P. T., D. P. R. Muller, and A. M. Lawson. 1982. The bile acid composition of gastric contents from neonates with high intestinal obstruction. Biochem. J. 206:489-498.

16. Setchell, K. D. R., A. M. Lawson, N. Tanida, and J. Sjövall. 1983. General methods for the analysis of metabolic profiles of bile acids and related compounds in faeces. J. Lipid Res. 24:1085-1100.

17. Setchell, K. D. R., and A. Matsui. 1983. Serum bile acid analysis. Clin. Chim. Acta. 127:1-17.

18. Norman, A., and B. Strandvik. 1971. Formation and metabolism of bile acids in extrahepatic biliary atresia. J. Lab. Clin. Med. 78:181-193.

19. Une, M., F. Nagai, K. Kihira, T. Kuramoto, and T. Hoshita. 1983. Synthesis of four diastereoisomers at carbons 24 and 25 of $3 \alpha, 7 \alpha, 12 \alpha, 24$-tetrahydroxy-5 $\beta$-cholestan-26-oic acid, intermediates of bile acid synthesis. J. Lipid Res. 24:924-929.

20. Ali, S. S., E. Stephenson, and W. H. Elliott. 1982. Bile acids, LXVII. The major bile acids of Varanus monitor. J. Lipid Res. 23:947-954.

21. Une, M., T. Kuramoto, and T. Hoshita. 1983. The minor bile acids of the toad, Bufo vulgaris formosus. J. Lipid Res. 24:1468-1474.

22. Lawson, A. M., and K. D. R. Setchell. 1987. Mass spectrometry of bile acids. In The Bile Acids. Vol. 4. Plenum Press, New York, London. Chapter 5.

23. Une, M., F. Nagai, and T. Hoshita. 1983. High performance liquid chromatographic separation of higher bile acids. J. Chromatogr. $257,411-415$.

24. Noma, Y., M. Une, K. Kihira, M. Yasuda, T. Kuramoto, and T. Hoshita. 1980. Bile acids and bile alcohols of the bullfrog. J. Lipid Res. 21:339-346.

25. Une, M., I. Morigami, K. Kihira, and T. Hoshita. 1984. Ste-

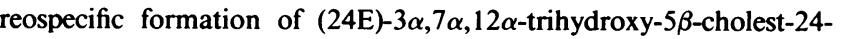
en-26-oic acid and (24R,25S)-3 $\alpha, 7 \alpha, 12 \alpha, 24$-tetrahydroxy-5 $\beta$-cholestan-26-oic acid from either (25R)- or (25S)-3 $\alpha, 7 \alpha, 12 \alpha$-trihydroxy- $5 \beta$ cholestan-26-oic acid by rat liver homogenate. J. Biochem. (Tokyo). 96:1103-1107.

26. Chu, C-h., and H. Schulz. 1985. 3-Hydroxyacyl-CoA epimerase is a peroxisomal enzyme and therefore not involved in mitochondrial fatty acid oxidation. FEBS (Fed. Eur. Biochem. Soc.) Lett. 185:129134.

27. Kärki, T., E. Hakkola, I. E. Hassinen, and J. K. Hiltunen. 1987. $\beta$-Oxidation of polyunsaturated fatty acids in peroxisomes. Subcellular distribution of $\Delta^{3}, \Delta^{2}$-enoyl-CoA isomerase activity in rat liver. FEBS (Fed. Eur. Biochem. Soc.) Lett. 215:228-232.

28. Karlaganis, G., V. Karlaganis, and J. Sjövall. 1984. Identification of 27 -nor- $5 \beta$-cholestane- $3 \alpha, 7 \alpha, 12 \alpha, 24,25,26$-hexol and partial characterisation of the bile alcohol profile in urine. J. Lipid Res. 25:693-702.

29. Wanders, R. J. A., C. W. T. van Roermund, A. Schelen, L. Ylst, R. B. H. Schutgens, and J. M. Tager. 1989. Peroxisomal bifunctional enzyme deficiency: a new type of peroxisomal $\beta$-oxidation defect with normal enzyme protein but deficient enzyme activity. Abstracts of the 27th Annual Symposium of the Society for the Study of Inborn Errors of Metabolism, Munich 1989. 154.

30. Watkins, P. A., W. A. Chen, C. J. Harris, G. Hoefler, S. Hoefler, D. C. Blake, Jr., A. Balfe, R. I. Kelley, A. B. Moser, M. E. Beard, and H. W. Moser. 1989. Peroxisomal bifunctional enzyme deficiency. $J$. Clin. Invest. 83:771-777. 